\title{
Identification of applicable patterns to algorithmization in BIM to explore solutions in the design stage of Social Housing
}

\section{SIGRADI2018} TECHNOPOLITICAS

xxii congresso da sociedade iberoamericana de gráfica digital 22th conference of the iberoamerican society of digital graphics 07|08|09|novembro|2018 iauusp | são carlos | spbr

\author{
Giovanna Tomczinski Novellini Brígitte \\ Universidade Estadual de Campinas | Brazil | giovanna.novellini@gmail.com \\ Regina Coeli Ruschel \\ Universidade Estadual de Campinas | Brazil | ruschel@g.unicamp.br
}

\begin{abstract}
In architecture, the algorithm application for innovative solutions exploration has become particularly important as the basis for distinct methodological approaches, becoming a significant technological artifact both for form exploration, as for the urban insertion and implantation. In this sense, the primary objective of this work is the identification, the description and the formulation of the algorithm that permeate patterns from Alexander (1977), for the context of social interest housing complexes project. It is wished to foment the possibility of incorporating the human-environmental relationship knowledge to the Building Information Modeling, through generative systems for the enhancement of creative practices.
\end{abstract}

Keywords: Algorithm; Patterns; Building Information Modelling; Computational design; Social housing.

\section{INTRODUÇÃO}

A baixa qualidade dos projetos de habitação de interesse social (HIS) no Brasil é frequentemente relacionada à uma metodologia desestruturada onde aspectos importantes do projeto, como as necessidades do cliente e a relação ser humano-ambiente, são negligenciados em função de outros. Ademais, constata-se o pouco uso da tecnologia de informação, de ferramentas automatizadas para análise de projetos, e até mesmo a aplicação de pesquisas capazes de subsidiar a solução de problemas, de monotonia e repetição geradas pela precariedade das soluções, retroalimentando o processo de projeto.

O cenário exposto faz emergir a necessidade de rediscutir aspectos levantados por movimentos como o Design Methods (1960) e o Design Thinking (1969), devido não somente à repercussão que tiveram, mas principalmente às importantes contribuições como da avaliação pósocupação, do programa arquitetônico, da inteligência artificial e da aplicação de técnicas computacionais para solucionar os problemas de projeto, e compor as formas dos objetos.

Da primeira abordagem sistemática, proposta por Jones (1963), ao interesse nos sistemas de processamento de informações e sistemas de suporte às decisões de projeto, importantes conceitos foram identificados e nos permitem compreender a complexidade do processo de projeto. Como, por exemplo, a compreensão da sequência de decisões composta pela análise, síntese e avaliação como parte de um processo de projeto flexível, posto de forma conjunta, articulado e com ciclos iterativos e influenciados por diversos agentes. Ou ainda, de que algoritmos matemáticos podem ser usados para direcionar inicialmente as relações e soluções, onde cada variante de projeto pode satisfazer cada requisito mínimo.

Neste sentido, estratégias paramétricas / algorítmicas têm se tornado particularmente importantes por servirem de base para distintas abordagens metodológicas (Meredith, 2008). Como apresenta Hudson (2010), o processo paramétrico de projeto é definido como o desenvolvimento de um modelo ou descrição de um problema, onde a representação é baseada nas relações entre objetos controlados por variáveis: os parâmetros.

Em suma, a modelagem paramétrica traz como potencial a dinâmica de alteração de parâmetros para auxílio na tomada de decisão que, quando aplicada com objetivo generativo, aumenta a probabilidade de ocorrências aleatórias, resultando num maior número de soluções. Uma das formas de se realizar este processo é através da utilização de ambientes de programação visual para modelagem. De acordo com Koniset al.(2016), o Grasshopper, utilizado com Rhino 3D, é uma VPL (visual programming language) comumente usada na indústria de construção, mas outros plug-ins como Dynamo (Autodesk, 2015) e Marionette (Vectorworks, 2015) estão se tornando mais proeminentes. Os dois últimos, assim como Generative Component para o AECOsim (da Bentley), permitem que modelos de concepção digital formativo, generativo e baseado em desempenho (Oxman, 2006), possam ser mediados por BIM, evitando assim o rompimento do fluxo de informação e permitindo o reuso do modelo entre as fases de concepção e desenvolvimento (Ruschel, 2014).

É comum observarmos a criação de algoritmos relacionados ao desempenho quantitativo (acústico, 
térmico, luminoso), mas não ao qualitativo (ser humanoambiente). Sendo assim, revisou-se a literatura, constatando em Alexander et al. (1977) e em sua sistematização criativa na solução de problemas do projeto através dos patterns, a capacidade de amparar a construção de algoritmos capazes de explorar novas soluções na concepção de projetos que enfoquem a relação ser humano-ambiente, através de ferramentas que permitem BIM.

A obra em questão consiste na coletânea de 253 patterns que, de acordo com suas relações, estabelecem uma rede formada por uma lista explicativa de situações recorrentes em projetos, caracterizadas por meio de patterns, que vêm acompanhadas de um exemplo arquetípico, na forma gráfica, que descreve o problema e a essência da solução (Van der Voordt \& Van Wegen, 2005). Os parâmetros identificados buscam contribuir para alcançar a qualidade sem nome, fenômeno identificado em seu livro The timeless way of building (Alexander, 1979).

Frente às criticas significativas recebidas pela publicação sobre o pouco conteúdo cientifico dos patterns, ou ainda a negação da legitimidade de um pluralismo de valores em arquitetura, autores como Barros \& Kowaltowski (2013) ressaltam a importância dos patterns em demonstrar uma conexão direta à vivência humana no ambiente construído, percebida por um senso comum e entendimento natural.

A iniciativa pioneira constatada por Barros \& Kowaltowski (2013) sobre A Pattern Language consiste na organização de dados da relação ser humano-ambiente para sustentar soluções projetuais, buscando ligar, de forma sistemática, o comportamento humano a elementos arquitetônicos. É esta característica que torna a obra relevante para a pesquisa em que este artigo se insere, uma vez que os patterns de projeto identificados podem contribuir como importante material de referência para a construção de algoritmos capazes de explorar novas soluções na concepção de projetos que enfoquem a relação ser humano-ambiente, através de ferramentas de modelagem da informação da construção.

Resgatar a metodologia ou as soluções propostas por Alexander permite-nos salientar as reflexões e diretrizes atuais de projeto levantadas por pesquisadores da qualidade ambiental (Barros \& Kowaltowski, 2013; Oliveira e Sousa \& Moreira, 2013) quanto à relação ambientecomportamento humano, especialmente no que diz respeito à qualidade e diversidade socioambiental.

O presente artigo apresenta o resultado parcial de uma pesquisa de doutorado em andamento, pelo Programa de Pós Graduação em Arquitetura, Tecnologia e Cidade FECUNICAMP, cujo objetivo principal é legitimar a aplicação de ferramentas que permitem BIM na etapa de concepção associado à Gramática da Forma Paramétrica, como metodologia de suporte ao processo de projeto. Esta proposição será validada através da aplicação do método no contexto do projeto de conjuntos habitacionais de interesse social, fomentando a possibilidade de incorporação do conhecimento referente à relação ser humano-ambiente ao BIM, através de sistemas generativos. Propondo-se, assim, aproximar dois ramos da arquitetura digital, isto é, o design computacional e a modelagem da informação da construção.
O principal objetivo do estudo exploratório que origina este artigo é a identificação, descrição gráfica e matemática dos algoritmos que permeiam os patterns, que futuramente serão aplicados nos sistemas generativos através da utilização de ambientes BIM de programação visual para modelagem na criação.

\section{METODOLOGIA}

A estrutura da metodologia dar-se-á a partir do método científico hipotético-dedutivo de Popper (2005), apresentado em seu livro The Logicof Scientific Discovery, devido ao caráter exploratório desta pesquisa experimental. Segundo Popper, partimos de um problema (ou situação problema), construímos tentativas de soluções (hipóteses, conjecturas, teorias) e por meio de avaliação crítica descartamos aquelas que não resolvem satisfatoriamente o problema.

Enquanto pesquisa exploratória, buscou-se por meio da pesquisa experimental familiarizar-se com o problema: em que medida os patterns podem contribuir na elaboração de algoritmos? Tornando-o mais explícito na constituição da hipótese. A hipótese considera que os patterns são aplicáveis na construção de algoritmos por possuírem recomendações explícitas, geométricas ou informacionais. No método de investigação adotado, houve um recorte restringindo à investigação de uma seleção de 74 patterns, identificada por Barros (2008).

Os patterns relacionados ao tema habitacional foram selecionados preliminarmente dentre os 253 descritos e pertencentes a quaisquer das três seções de Alexander et al. (1977). No decorrer da pesquisa, durante a avaliação dos projetos, a seleção inicial foi sendo lapidada, com o acréscimo ou eliminação de alguns patterns, buscando-se extrair destes o conteúdo relevante para aquela avaliação quanto ao melhor atendimento de necessidades psicossociais e ambientais (Barros, 2008). A nova coletânea de patterns (74) foi organizada e apresentada em duas escalas que compõem a proposta de estratégia metodológica de apoio ao processo projetual: Senso de Urbanidade (37) e Senso de Habitabilidade (45), importante neste momento ressaltar que um determinado pattern pode fazer parte de um ou ambos os grupos.

Destaca-se, a seguir, os principais procedimentos adotados nesta pesquisa: (01) Classificação dos patterns quanto ao LOD (Leve of Development), dentro das escalas de aplicação: Senso de Urbanidade e Senso de Habitabilidade; (02) Identificação das categorias de recomendação: (i) explícita (objetivo) ou implícita (subjetivo), (ii) geométrica ou informacional (Solihin \& Eastman, 2015); (03) Identificação das frases/parágrafos que contém as variáveis; (04) Elaboração do fluxograma de cada algoritmo, explicando graficamente o conjunto de passos planejados para que se alcance o propósito do pattern; (05) Descrição das sentenças matemáticas, identificando, quando aplicável, os tipos de parâmetros e sua inter-relação.

\section{RESULTADOS}

O nível de desenvolvimento (LOD) descreve o grau de completude que um elemento do modelo de informação é 

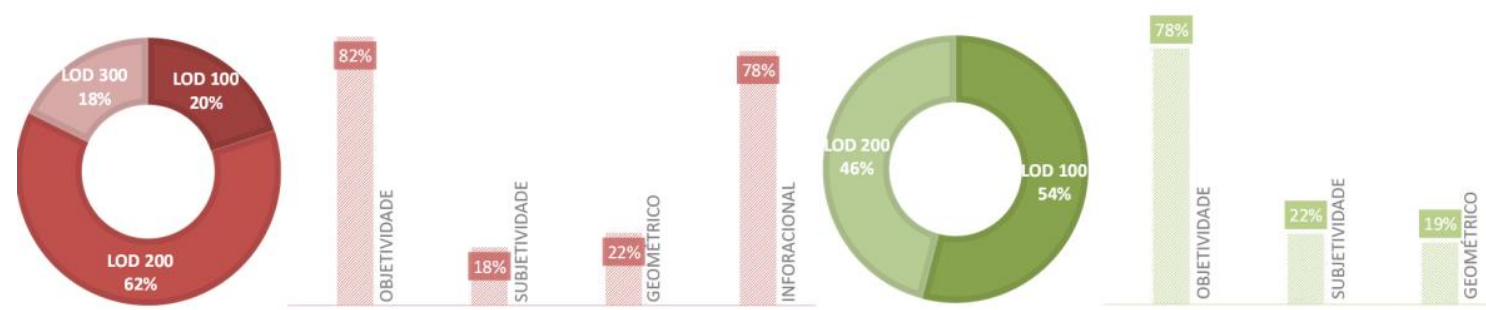

Figura 1: classificação dos patterns quanto ao LOD e identificação das categorias de recomendação. Fonte: Autores.

desenvolvido, explicitando os dados mínimos dimensionais, espaciais, quantitativos e qualitativos. Os LODs são categorizados em: Conceitual (LOD 100), Geometria Aproximada (LOD 200), Geometria Precisa (LOD 300), Fabricação (LOD 400) e As Built (LOD 500). Desta classificação delimitaremos os patterns que serão aplicados na etapa de concepção aos que corresponderem ao LOD 200.

A Figura 1 apresenta a análise dos 74 patterns que permitiu não apenas classificá-los quanto ao LOD dentro as escalas de aplicação: Senso de Urbanidade e Senso de Habitabilidade, como também quanto às categorias de recomendação, se explícita (objetivo) ou implícita (subjetivo) e geométrica ou informacional. Desta classificação foram considerados passíveis de parametrização 35 patterns (objetivo e geométrico ou objetivo e informacional). A seguir, no Quadro 1, têm-se os 35 patterns, agrupados por escala de aplicação, organizados em ordem alfabética (em sua tradução) e seguidos da numeração adotada na publicação original.

Quadro 1:Patterns parametrizáveis dentro da escala de aplicação

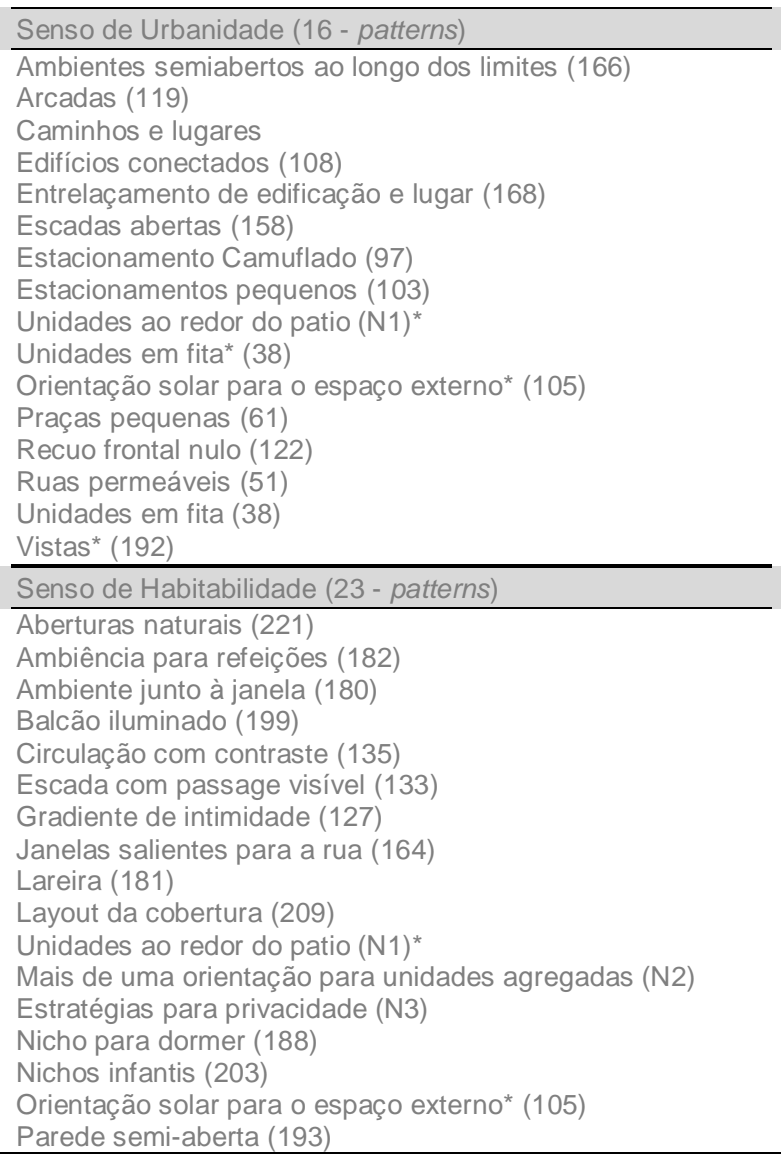

Posição e luz (128)

Unidades em fita* $(38)$

Veranda utilizável (167)

Variação de pé-direito (190)

Vistas* $^{*}(192)$

Zonas de piso (233)

A seguir apresenta-se a proposta de algoritmização de 3 patterns distintos entre si mas representativos dentre os parametrizáveis (Quadro 1). Inicia-se pela identificação do pattern através do seu nome seguido da frase com a solução proposta, nesta destacam-se as frases/parágrafos com as variáveis que foram identificadas. Posteriormente descrevemos matematicamente a sentença resultante do algoritmo ilustrado pelo fluxograma, através das linguagens matemáticas explicitadas por Bianconi (2002): (1) proposicional, aplicando as conjunções "e" $(\wedge)$, "ou" $(\vee)$ e a relação de implicação: que pode ser descrita por $\Rightarrow$ ou ainda, se A, então B; e a (2) quantificação, aplicando os operadores da matemática básica (,$+=, \cap$, entre outros).

O primeiro pattern a ser apresentado é o Zonas de pisos (233-floor surface): "Crie zonas pública e íntima, correspondendo respectivamente a materiais resistentes e macios. Marque claramente a transição entre elas." (grifo nosso)

$$
Z p A c \wedge Z p A p=A s+A m ; Z p A s \cap Z p A m=s l
$$

Onde: $Z p A c=Z$ Zna de piso em área comum; $Z p A p=Z$ Zona de piso em área privada; $A s=$ Área seca; $A m=$ Área molhada; sl=soleira.

Para facilitar a compreensão a Figura 2 apresenta o fluxo do algoritmo proposto para Zonas de piso.

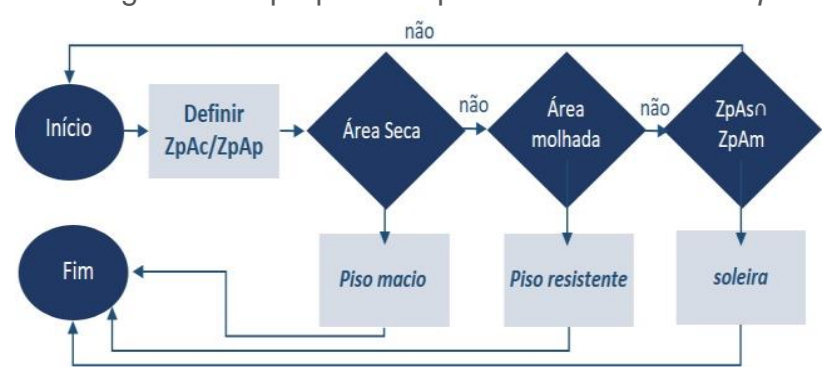

Figura 2: Zonas de pisos: fluxograma. Fonte: Autores.

O segundo pattern a ser apresentado é o Gradiente de intimidade (127-intimacy gradient): "Arranje os ambientes da UH de modo a criar sequência que comece pelas partes mais públicas e se encaminhe para áreas um pouco mais privadas, finalizando com os domínios mais íntimos." (grifo nosso). 


$$
\operatorname{GrIt}(U H) \Rightarrow S l \rightarrow(C z \wedge A s) \vee B h \rightarrow Q t
$$

Onde: GtIt $=$ Gradiente de intimidade; $U H=$ Unidade habitacional; $S I=$ Sala; $C z=$ Cozinha; $A s=$ Área de Serviço;Bh= Banheiro; $Q t=$ Quarto.

Apresenta-se a seguir através da Figura 3 o fluxo proposto para o algoritmo Grau de intimidade.

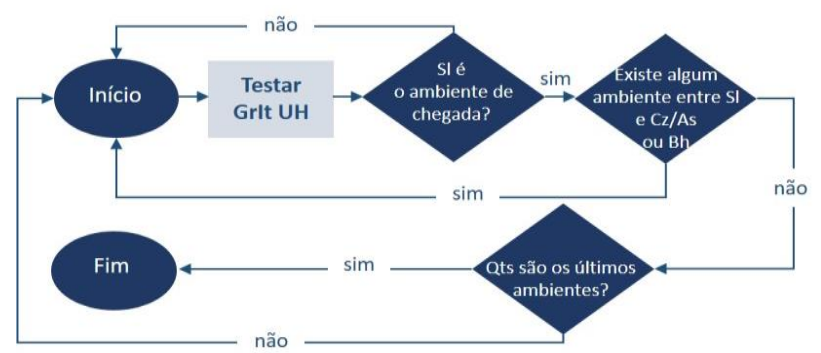

Figura 3: Gradiente de intimidade: fluxograma. Fonte: Autores.

O terceiro pattern a ser apresentado é o Edifícios conectados (108-connectedbuildings): "Onde possível, conectar edifícios novos aos existentes. Não manter recuos entre eles, mas formá-los como continuações." (grifo nosso). Tem-se o fluxo do algoritmo ilustrado pela Figura 4.

$$
E d E x \Rightarrow r c l=0(P s E f ; E d E x)
$$

Onde: $E d E x=$ Edificação Existente; $r c l=$ recuo lateral; PsEdf = Posição da Edificação.

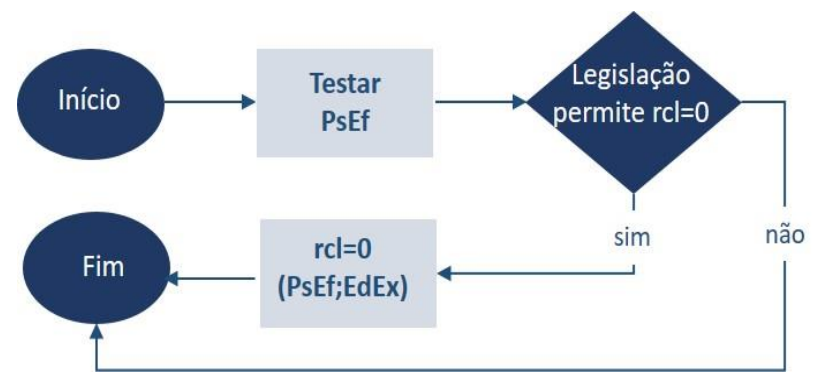

Figura 4: Edifícios conectados: fluxograma. Fonte: Autores.

Apresentamos a associação dos algoritmos ao BIM adaptando o modelo sugerido por Chen et al. (2011). No modelo proposto, a relação dos atributos é estabelecida em três aspectos: (A) classificação do objeto, (B) dados geométricos do objeto e $(C)$ informação da construção, conforme ilustrado na Figura 5.
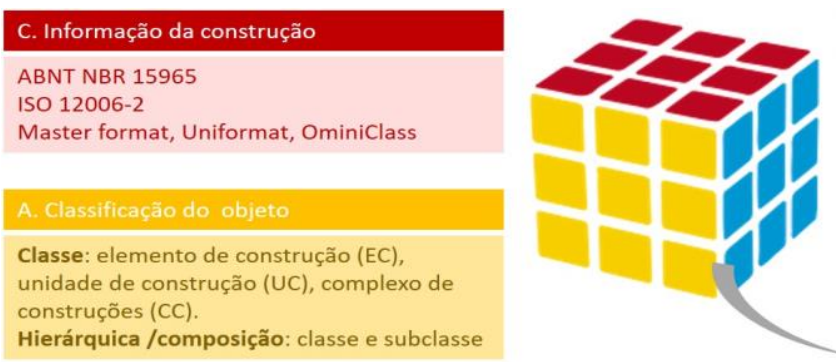

A classificação do objeto está relacionada à classe que pertence: elemento de construção (EC), unidade de construção (UC) ou complexo de construções (CC), ou ainda, a sua classificação hierárquica e/ou de composição. Os dados geométricos do objeto relacionam-se diretamente à sua modelagem (coordenadas, dimensões e relações que estabelecem com objetos/ componentes adjacentes). Já para a classificação da informação no BIM, seguimos os conceitos básicos adotados pela ABNT NBR 15965 - Sistema de classificação da informação da construção, norma nacional correlacionada com a classificação OMNICLASS.

Vale ressaltar que a classificação da informação é fundamental por contribuir em diversas áreas e etapas do processo de projeto garantindo assertividade e interoperabilidade, podendo ser feita através de diferentes sistemas de classificação, dentre os principais apresentados por Silva \& Amorim (2011) estão o Masterformat (hierárquica), o Uniformat (facetadas) ou ainda, o já citado OMINICLASS (hierárquicas e facetadas).

No cenário nacional, vemos essa preocupação refletida nos esforços empenhados pela comissão de estudos da ABNT, a CEE-134 - Comissão de Estudo Especial de Modelagem de Informação da Construção (BIM), cujo trabalho resultou na publicação das seguintes normas: ABNT NBR ISO 12006-2:2010 Construção de edificaçãoOrganização de informação da construção - Parte 2: Estrutura para classificação e ABNT NBR 15965-Sistema de classificação da informação da construção. A primeira refere-se a uma tradução da ISO 12006-2 (2001), atualmente revisada para ISO 12006-6:2015, mas ainda não traduzida para o português. Esta norma apresenta uma estrutura geral para os sistemas de classificação da construção. Já a segunda, têm publicadas quatro das sete partes previstas cujo conjunto, quando completo, será responsável por descrever em 13 tabelas uma classe da construção. A saber: Parte 1: Terminologia e estrutura (2011); Parte 2: Características dos objetos da construção (2012) - tabelas 0M-Materiais e 0P-Propriedades, Parte 3: Processos da construção (2014) - tabelas 1F-Fases, 1SServiços e 1D-Disciplinas e Parte 7: Informação da construção (2015) - tabela 5I-Informação. Das três partes restantes, a Parte 6: Unidades da construção ainda não foi publicada, mas, já teve o seu texto aprovado pela comissão - tabelas 4U-Unidades e 4A-Espaços. Restando, apenas a Parte 4: Recursos da construção tabelas 2N-Funções Organizacionais, 2Q-Equipamentos e 2C-Componentes e Parte 5: Resultados da construção tabelas: 3E-Eementos e 3R-Resultados da Construção (ABDIMDIC, 2017).

Figura 5: atributos do modelo BIM. Fonte: Autores. 


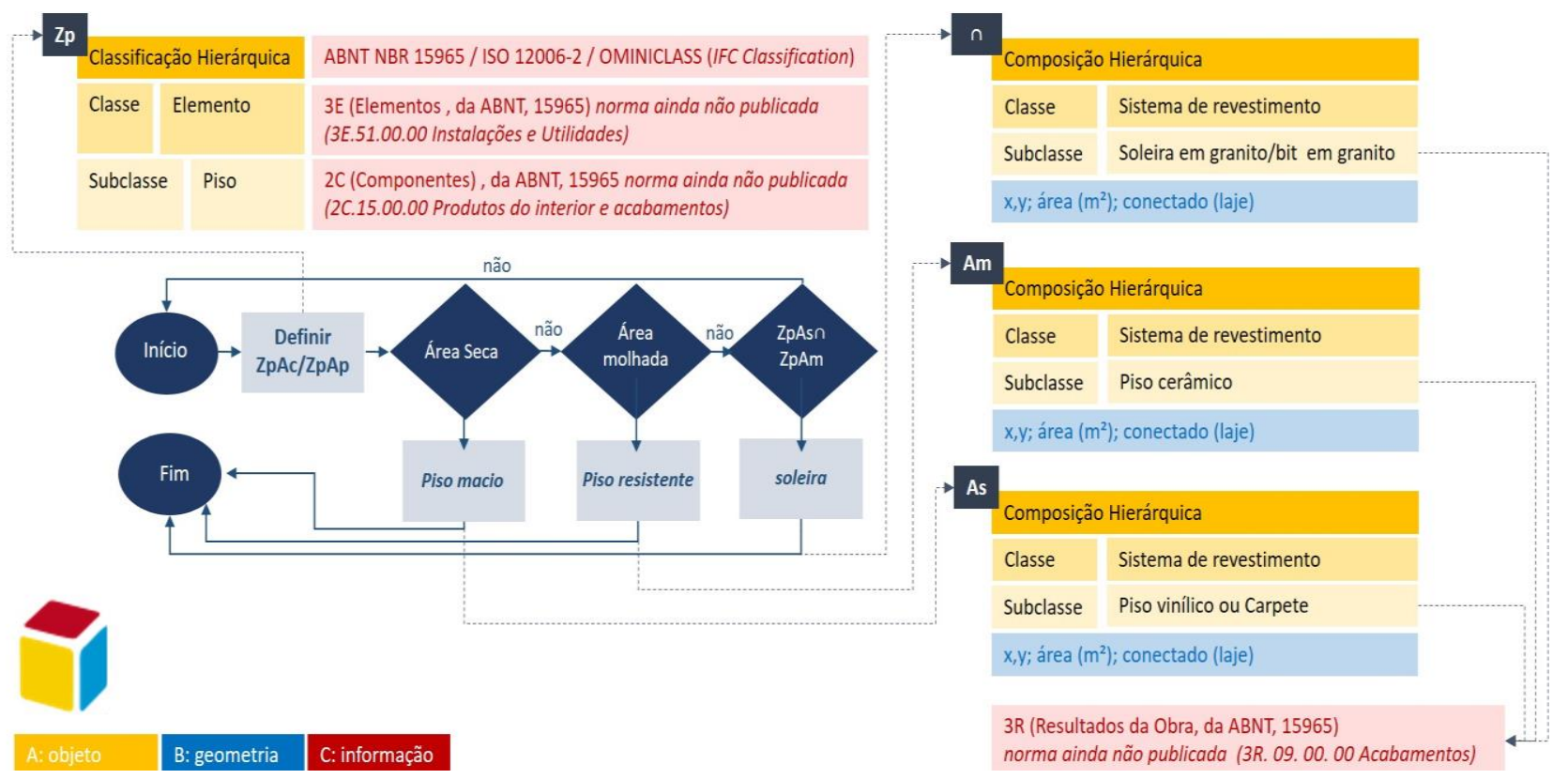

Figura 6: Zonas de pisos: classificação BIM. Fonte: Autores.

Por fim, a Figura 6 é apresentada como um exemplo da associação dos algoritmos ao modelo BIM. Para cada um dos 35 patterns identificados tem-se, a partir do fluxo proposto para o algoritmo, a identificação das variáveis como atributos em três aspectos: (A) classificação do objeto, (B) dados geométricos do objeto e (C) informação da construção, através do sistema proposto pela $A B N T$ NBR 15965-Sistema de classificação da informação da construção. Tal classificação permitirá identificar possíveis relações de interdependência entre os patterns, ou seja, na variação de uma determinada variável/atributo, tendo por base um determinado pattern, a aplicação de outro será de alguma forma afetada.

\section{CONSIDERAÇÕES FINAIS}

Resgatar e rediscutir aspectos levantados por movimentos como o Design Methods (1960) e o Design Thinking (1969) torna-se eminente frente à produção da habitação de interesse social, frequentemente associada à homogeneização das soluções projetuais, replicadas em diferentes estados brasileiros, independente das necessidades locais e dos próprios usuários. Tal prática não tem se mostrado capaz de garantir a qualidade dos projetos, nem de incorporar as reais necessidades dos beneficiários.

Neste sentido, este artigo se propôs a identificar os patterns aplicáveis à algoritmização que fossem capazes de explorar novas soluções na concepção de projetos que enfoquem a relação ser humano-ambiente, através de ferramentas que permitem BIM. A partir da revisão bibliográfica foram utilizados 74 patterns para esta avaliação. Destes, foram identificados 35 algoritmos, dos 41 que se inserem no LOD 200, que poderão ser explorados na próxima etapa da pesquisa de doutorado, onde este estudo se insere.

Durante a pesquisa exploratória desenvolvida neste estudo observou-se que um fator complicador pode ser a subjetividade durante a criação dos algoritmos. No entanto, a contribuição do procedimento de algoritmização de dados da relação ser humano-ambiente para sustentar soluções projetuais, buscando ligar, de forma sistemática, o comportamento humano a elementos arquitetônicos, pode ser muito útil para auxiliar o processo de projeto, apresentando-se assim como um importante campo a ser explorado.

Parte-se da ideia de que uma solução de projeto é composta pela integração entre parâmetros, portanto, não podemos deixar de considerar os aspectos qualitativos além dos quantitativos.

Como continuidade a esta etapa da pesquisa, propõem-se o desenvolvimento de uma árvore de decisão contendo a integração dos 35 algoritmos na elaboração de um projeto de HIS. A identificação dos parâmetros e algoritmos associados à relação ser humano-ambiente nos permitirá sínteses e avaliações mais dinâmicas, capazes de retroalimentar o processo de projeto orientando-o às decisões mais qualificadas para a solução de problemas sem que, no entanto, esta desconsidere tais relações.

O processo de algoritmização e sua associação ao sistema de classificação BIM, proposto por este trabalho, abre caminho para a elaboração de algoritmos voltados não apenas à HIS, como também a outras tipologias de edifício, a partir de dados que podem ser comparados e verificados entre as publicações como as de Alexander, et al (1977), Barros (2008), Oliveira e Sousa \& Moreira (2013). Ademais, permite que processos pparticipativos possam servir de estruturas iniciais ao desenvolvimento de novos algoritmos.

\section{REFERÊNCIAS}

ABDI-MDIC (2017). Projeto Guias Técnicos BIM - Edificações. Guia 2 - Classificação da informação no BIM. Brasília-DF: Agência Brasileira de Desenvolvimento Industrial, 2017.

Alexander, C., Ishikawa, S. \& Silverstein, M.(1977). A Pattern Language: Towns, Buildings, Construction. New York: NY: Oxford University Press.

Alexander, C. (1979). The timeless way off building. New York: NY: Oxford University Press. 
Barros, R. (2008). Habitação coletiva: a inclusão de conceitos humanizadores no processo de projeto. Ph.D. thesis, Universidade Estadual de Campinas.

Barros, R., Kowaltowski, D. (2013). Do projeto urbano ao detalhe construtivo. A PatternLanguage finalmente traduzida. Resenhas Online, 12, n.137.01, Vitruvius. Retrievedfrom http://www.vitruvius.com.br/revistas/read/resenhasonline/12.1 $37 / 4734$

Bianconi, R. A linguagem matemática. Classnotes, IME-USP, 2002. Disponível em: https://www.ime.usp.br/ bianconi/recursos/lo.pdf. Acesso em: 18 de mai.de2018.

Chen, Y. J., Feng, C. W., Wang, Y. R., Wu, H. M. (2011) Using bim model and genectic algorithms to optimize the crew assignment for construction project planning, International Journal of Technology, 2, 3, 179-188.

Hudson, R. (2010). Strategies for Parametric Design in Architecture: An application of led research. Ph.D. thesis, University of Bath.

Jones, J. C. (1963). Method of Systematic Design. Conference on design, Londres: Pergamon Press, 53-73.

Konis, K, Gamas, A \& Kensek, K. (2016). Passive performance and building form: Anoptimization framework for early-stage design support. Solar Energy, 125, 161-179.
Meredith, M. (2008). From Control to Design: Parametric/Algorithmic Architecture. NY: Actar-D.

Oliveira e Sousa, M. N. P., Moreira, D. C. (2013). Aplicação da linguagem de padrões à avaliação de projetos de biblioteca pública. Gestão e Tecnologia de Projetos, São Paulo, v. 8, n. 2, p. 7-25, jul./dez. 2013. http://dx.doi.org/10.11606/gtp. v8i2.80946.

Oxman, R. (2006). Theory and design in the first digital age. Design Studies, 27, 229-265.

Ruschel, R. C. (2014). To BIM or not to BIM. III Encontro da Associação Nacional de Pesquisa e Pós-Graduação em Arquitetura e Urbanismo. Anais...São Paulo: ENEPARQ.

Silva, J. C. B., Amorim,S. R. L. () A Contribuição dos Sistemas de Classificação Para a Tecnologia BIM: uma abordagem teórica. Encontro de Tecnologia de informação e comunicação na construção, 5 .

Solihin, W., \& Eastman, C. (2015). Classification of rules for automated BIM rule checking development. Automation in construction, 53, 69-82.

Van der Voordt, D. J. M., VanWegen, H. R. (2005). Architecture in use: an introduction to the programming, design and evaluation of buildings. Routledge. 